\title{
Complete Monte Carlo RF Analysis of "Real" Short-Channel Compound FET's
}

\author{
Sharief Babiker, Asen Asenov, Nigel Cameron, Steven P. Beaumont, and John R. Barker
}

\begin{abstract}
A comprehensive RF analysis technique based on ensemble Monte Carlo (EMC) simulation of compound FET's with realistic device geometry is presented. $Y$-parameters are obtained through Fourier transformation of the EMC transients in response to small changes in the terminal voltages. The terminal currents are statistically enhanced and filtered to allow for reliable $y$-parameters extraction. Improved analytic procedure for extracting the intrinsic device small-signal circuit components is described. As a result, stable $y$-parameters and reliable circuit components can be extracted for the whole range of device operation voltages. Parasitic components like contact and gate resistances are included in the $y$-parameters at a post processing stage to facilitate the forecast of the performance figures of merit of real devices. The developed RF technique has been applied in the EMC simulation of pseudomorphic HEMT's (pHEMT's) fabricated at the Glasgow Nanoelectronics Research Center. Good agreement has been achieved between the simulated and measured small-signal circuit components and performance figures of merit.
\end{abstract}

Index Terms - Compound FET's, Monte-Carlo, RF analysis, simulation.

\section{INTRODUCTION}

C OMPOUND FET's such as GaAs MESFET's and InGaAs HEMT's with channel lengths approaching $0.1 \mu \mathrm{m}$ have shown significant potential for microwave applications up to W-band [1]. To a great extend this is due to the a well pronounced velocity overshoot in the high field region of the channel. Here, the carrier velocity in the above materials may exceed saturation velocity by several times [2]. In many cases, the predictive analysis and computer-aided design of such devices require full scale ensemble Monte Carlo (EMC) simulation. In a series of EMC simulation studies, the nonstationary transport in GaAs MESFET's [3], InP MESFET's [4], AlGaAs/GaAs HEMT's [5]-[7], and InGaAs pseudomorphic HEMT's (pHEMT's) [8], [9] has been investigated. Most of the published papers, however, consider simplified device geometry and focus mainly on the transport physics and the effect of the enhanced channel velocity on the DC characteristics. Of more importance for the proper design of modern shortchannel compound FET's is the RF performance. This is determined not only by the high field transport but also by device geometry, surface effects, and contact resistance [10].

Manuscript received October 31, 1997. The review of this paper was arranged by Editor W. Weber. This work was supported by EPSRC through the Nanoelectronics Rolling Grant RG/6601BS.

The authors are with the Device Modeling Group and Nanoelectronics Research Center, Department of Electronics and Electrical Engineering, University of Glasgow, Glasgow G12 8LT, Scotland, U.K.

Publisher Item Identifier S 0018-9383(98)05338-6.
The $T$ - or $\Gamma$-shaped gate, gate recess, and passivation in such devices critically affect the device parasitics and the overall RF performance.

Although the fundamentals of the RF and noise EMC analysis are outlined in [11], very few Monte Carlo compound FET simulation papers address the RF performance issues in depth. In [12], a strategy for extracting the $y$-parameters and the small-signal equivalent circuit components from Monte Carlo simulation of GaAs MESFET's was presented. However, this analysis is restricted to simple rectilinear device geometries and low voltages and does not take into account the "intrinsic" source and drain series resistances presented in the simulations nor the "extrinsic" contact and gate resistances presented in a real device. Similar are the restrictions of the $\mathrm{RF}$ and noise EMC analysis of GaAs MESFET's in [13] and AlGaAs/GaAs HEMT's in [14]. A simplified simulation geometry usually underestimates the capacitive components and seriously affects the accuracy of the extracted small-signal circuit. This, together with the omission of the gate and the contact resistance in Monte Carlo simulations, does not allow realistic extraction of important RF figures of merit such as the cutoff frequency $f_{T}$ and the maximum frequency of oscillation $f_{\text {max }}$.

In this paper, we describe a comprehensive methodology to extract the RF performance of "real" compound FET's from time domain EMC simulation which can be used for practical device design. The methodology is based on transient finite element EMC simulation of realistic device geometries carried out using the Monte Carlo module of the heterojunction compound two-dimensional (2-D) finite element simulator $\mathrm{H} 2 \mathrm{~F}$ [15]. A proper description of the shape of the gate, recess, and source and drain contacts provides reliable values for the small-signal circuit components. The post processing inclusion of the contact and gate resistances and eventually inductive components in the extracted $y$-parameters allow for a realistic estimation of the RF performance of the compound FET's under investigation.

In the next section we outline the flow diagram of the complete RF EMC analysis of compound FET's. This includes the extraction of the small-signal equivalent circuit and the RF figures of merit. Sections III and IV deal with specific issues related to the reliable extraction of the frequency dependent two-port $y$-parameters from transient EMC simulation results. This forms the basis of the RF analysis. In Section V, the results of the complete RF EMC analysis of pHEMT's fabricated at the Glasgow Nanoelectronics Research Center are compared 
to the small-signal circuit components $f_{T}$ and $f_{\max }$, extracted from $s$-parameter measurements.

\section{The COMPLETE RF ANALYSIS}

The flow diagram of the complete time domain RF EMC analysis is given in Fig. 1. The analysis begins with the extraction of complex frequency dependent two-port $y$-parameters of the simulated compound FET. The $y$-parameters are calculated by Fourier decomposition of the current transients obtained in response to step perturbations in the terminal voltages. For a given dc working point $\left(V_{g}, V_{d}\right)$ a steady-state condition is first established by allowing the EMC simulation to run for a time long enough to achieve a steady average drain current $I_{d}(0)$. Then small step perturbations $\Delta V_{j}$ are applied to the gate and drain electrodes consecutively and the transient currents $I_{i}(t)$ are recorded as functions of time with the indices $i$, $j=1$ referring to the gate and $i, j=2$ referring to the drain electrode. The real and imaginary components of the $y$-parameters are given by the following transformations [16]:

$$
\begin{aligned}
\operatorname{Re}\left(Y_{\mathrm{ij}}^{\operatorname{sim}}(\omega)\right)= & \frac{I_{i}(\infty)-I_{i}(0)}{\Delta V_{j}} \\
& +\frac{\omega}{\Delta V_{j}} \int_{0}^{T}\left[I_{i}(t)-I_{i}(\infty)\right] \cdot \sin (\omega t) d t \\
\operatorname{Im}\left(Y_{\mathrm{ij}}^{\operatorname{sim}}(\omega)\right)= & \frac{\omega}{\Delta V_{j}} \int_{0}^{T}\left[I_{i}(t)-I_{i}(\infty)\right] \cdot \cos (\omega t) d t
\end{aligned}
$$

where $I_{i}(0)$ and $I_{i}(\infty)$ are the steady-state dc levels of the gate and the drain currents before and after the step perturbation, respectively. The recording time, $T$, includes the transient time which is typically a couple of picoseconds plus some additional simulation time during which enough statistics are accumulated for the steady-state post-transient current $I_{i}(\infty)$.

A proper estimation of the transient current immediately after the perturbation is vital to the correct extraction of the $y$-parameters. The highest frequency of interest $f_{h}$ determines the upper bound $\Delta t$ of the time step during the first few steps of the transient $\Delta t \ll\left(2 \pi f_{h}\right)^{-1}$. In modern compound FET's, $f_{h}$ may be several hundred gigahertz [17]. The amplitude of the displacement current spike associated with the feed back capacitances at $t=0^{+}$is proportional to $(\Delta t)^{-1}$. The lower bound on $\Delta t$ imposed in drift-diffusion transient simulations to prevent the loss of current continuity as a result of the finite computer word length [16] can be relaxed in Monte Carlo transient simulations which do not suffer from such problems. In this study we employ time steps of $\Delta t=0.125-1.000 \mathrm{fs}$ to resolve the beginning of the transient. This compares with the values of field adjusting time step $\Delta t=1-2.5$ fs used to achieve the initial steady state.

The magnitude of the perturbation $\Delta V_{j}$ should also be carefully chosen. It should be large enough to allow proper resolution of the transients bearing in mind the stochastic nature of the EMC current. At the same time, the perturbation must be small enough to avoid the generation of harmonics during the transient. Typical values of $100-200 \mathrm{mV}$ were used for the gate voltage perturbation $\Delta V_{1}$. Higher values of 200-300 mV are needed for the drain voltage perturbation $\Delta V_{2}$, especially in the saturation region of the FET operation.

\section{$\mathrm{RF}$ analysis of $\mathrm{MC}$ transients}

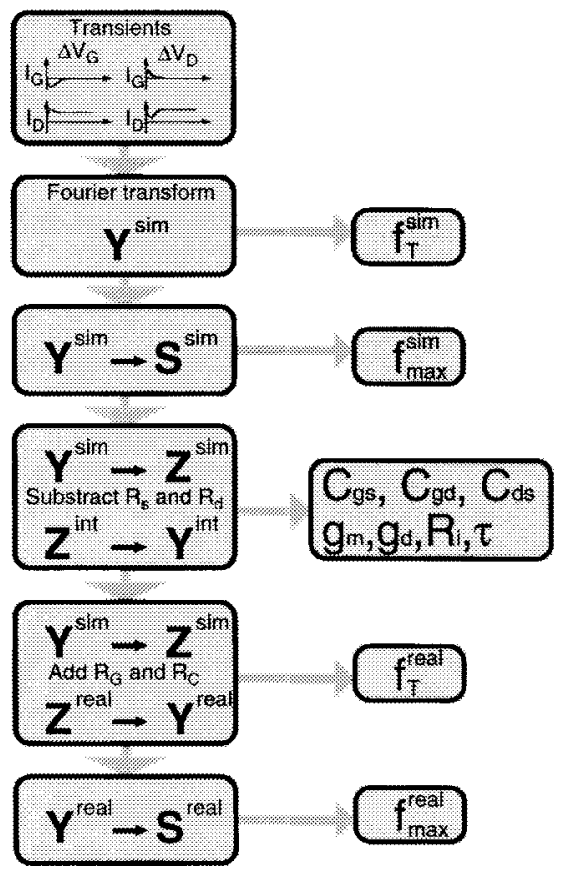

Fig. 1. Flow diagram of the RF EMC analysis.

Although these values are higher than the values recommended in [16], no appreciable generation of harmonics was observed due to the good linearity of the simulated devices.

Further details about the EMC simulation and the calculation of the terminal currents during the transient are given in Section III. The extracted $y$-parameters properly represent the geometry and surface effects but do not incorporate the contact and the gate resistances which are excluded from the EMC simulation. Inductive elements associated with the metal pads in a real device are also excluded from the simulation.

To proceed further with the RF analysis, the current gain $G_{c}=d I_{d} / d I_{g}$ is presented in terms of the extracted $y$ parameters as

$$
G_{c}^{\operatorname{sim}}=\left[\frac{\partial I_{d}}{\partial V_{g}} / \frac{\partial I_{g}}{\partial V_{g}}\right]=\left|y_{21}^{\operatorname{sim}} / y_{11}^{\operatorname{sim}}\right|
$$

The cutoff frequency of the simulated device $f_{T}^{\text {sim }}$ is extracted by solving $\log \left[G_{c}^{\operatorname{sim}}(\log f)\right]=0$ which is an almost linear function.

In order to extract the maximum frequency of oscillation of the simulated device $f_{\max }^{\operatorname{sim}}$ the $y$-parameters are transformed into the $s$-parameters $\mathrm{S}^{\mathrm{sim}}$. In terms of $s$-parameters the maximum stable gain, $G_{\mathrm{MSG}}^{\mathrm{sim}}$, and the maximum available gain, $G_{\mathrm{MAG}}^{\mathrm{sim}}$, are expressed by

$$
\begin{aligned}
G_{\mathrm{MSG}}^{\operatorname{sim}} & =\left|\frac{s_{21}^{\operatorname{sim}}}{s_{12}^{\operatorname{sim}}}\right| \quad \text { if } K^{\mathrm{sim}} \leq 1 \\
G_{\mathrm{MAG}}^{\operatorname{sim}} & =\left|\frac{s_{21}^{\operatorname{sim}}}{s_{12}^{\operatorname{sim}}}\right|\left(K^{\operatorname{sim}}-\sqrt{\left(K^{\operatorname{sim}}\right)^{2}-1}\right) \quad \text { if } K^{\mathrm{sim}}>1
\end{aligned}
$$




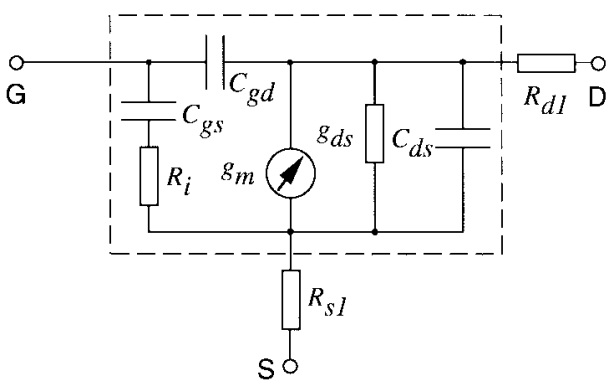

Fig. 2. Small-signal equivalent circuit model of the FET. Dashed lines enclose the equivalent circuit of the intrinsic device.

where

$K^{\mathrm{sim}}=\frac{1+\left|s_{11}^{\operatorname{sim}} s_{22}^{\operatorname{sim}}-s_{12}^{\operatorname{sim}} s_{21}^{\operatorname{sim}}\right|^{2}-\left|s_{11}^{\operatorname{sim}}\right|^{2}-\left|s_{22}^{\operatorname{sim}}\right|^{2}}{2\left|s_{12}^{\operatorname{sim}} s_{21}^{\operatorname{sim}}\right|}$

is the Rollette's stability factor. The maximum frequency of oscillation $f_{\max }^{\mathrm{sim}}$ is then extracted by solving $\log \left[G_{\mathrm{MAG}}^{\operatorname{sim}}(\log f)\right]=0$ which is also a well-behaved monotonously decreasing function.

The next analysis step is the extraction of the of the smallsignal equivalent circuit components. For typical MESFET and HEMT simulation domains, we have adopted the equivalent circuit presented in Fig. 2. The gate resistance, the contact resistances, and any external inductive components are excluded from the EMC simulation and hence from this circuit. The source $R_{s 1}$ and the drain $R_{d 1}$ resistances in Fig. 2 represent the resistance of the regions between the gate and the source and drain ohmic contacts, respectively. These resistances are included in the solution domain and can be evaluated directly from the EMC steady state simulation results prior to transients as the ratio of the voltage drop between the gate edge and the corresponding contact and the average current flowing through the device. Then the $y$-parameters of the simulated device $\mathbf{Y}^{\operatorname{sim}}$ are transformed into $z$-parameters $\mathbf{Z}^{\text {sim }}$. The estimated source and drain resistances $R_{s 1}$ and $R_{d 1}$ are subtracted from $\mathbf{Z}^{\text {sim }}$ to obtain the $z$-parameters $\mathbf{Z}^{\text {int }}$ of the "intrinsic" device equivalent circuit surrounded by dashed lines in Fig. 2

$$
\mathbf{Z}^{\mathrm{int}}=\mathbf{Z}^{\mathrm{sim}}-\mathbf{Z}^{\text {rd1 }}
$$

where

$$
\mathbf{Z}^{r d 1}=\left[\begin{array}{cc}
R_{s 1} & R_{s 1} \\
R_{s 1} & R_{s 1}+R_{d 1}
\end{array}\right]
$$

Finally, $\mathbf{Z}^{\text {int }}$ are transformed back into $y$-parameters $\mathbf{Y}^{\text {int }}$ from which the components of the "intrinsic" small-signal circuit can be analytically extracted [12].

The adopted equivalent circuit of a "real" device is also shown in Fig. 3. In order to evaluate the cutoff frequency $f_{T}^{\text {real }}$ and the maximum frequency of oscillations $f_{\max }^{\text {real }}$ of the "real" device, the gate resistance $R_{g}$, the contact resistances $R_{c}$, and eventually the inductive components $L_{g}, L_{s}$, and $L_{d}$ first have to be incorporated in the $z$-parameters of the real device $\mathbf{Z}^{\text {real }}$ [19]

$$
\mathbf{Z}^{\text {real }}=\mathbf{Z}^{\text {sim }}+\mathbf{Z}^{\text {gcl }}
$$

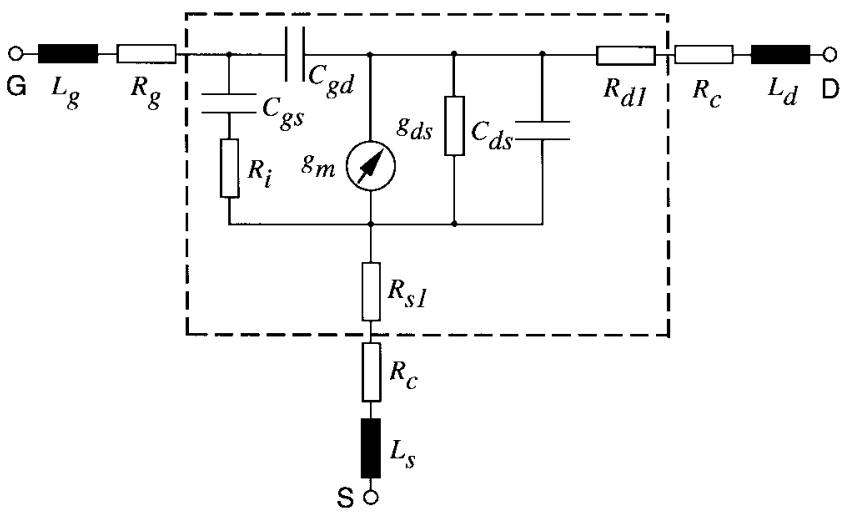

Fig. 3. Small-signal equivalent circuit model of the real FET. Dashed lines enclose the equivalent circuit model of the simulated FET.

where

$\mathbf{Z}^{g c l}=\left[\begin{array}{cc}\left(R_{g}+R_{c}\right)+j \omega\left(L_{g}+L_{s}\right) & R_{c}+j \omega L_{s} \\ R_{c}+j \omega L_{s} & 2 R_{c}+j \omega\left(L_{d}+L_{s}\right)\end{array}\right]$.

$\mathbf{Z}^{\text {real }}$ are then transformed into $\mathbf{Y}^{\text {real }}$. The value of $f_{T}^{\text {real }}$ is extracted by solving $\log \left[G_{c}^{\text {real }}(\log f)\right]=0$, where $\mathbf{G}_{c}^{\text {real }}$ is given by an expression analogous to (1). Finally, $\mathbf{Y}^{\text {real }}$ are transformed into $\mathbf{S}^{\text {real }}$ and $f_{\max }^{\text {real }}$ is extracted by solving $\log \left[G_{\mathrm{MAG}}^{\mathrm{real}}(\log f)\right]=0$ where $G_{\mathrm{MAG}}^{\text {real }}$ is given by an expression similar to (4) and (5). It must be noted that the inclusion of the contact resistances changes the dc working point of the "real" device to $\left(V_{g}+I_{d} R_{c}, V_{d}+2 I_{d} R_{c}\right)$ [20].

The EMC simulation provides a unique opportunity to investigate $a b$ initio the RF noise performance of compound FET's. However, the noise analysis is restricted to the simulated device and reflects only the noise generated by the carriers inside the device. In this study, the intrinsic minimum noise figure is calculated from the small-signal equivalent circuit model using the scheme presented in [21]. The noise sources associated with the ohmic contacts, the external circuit, and the measurement equipment are excluded from the analysis.

\section{Monte Carlo Transient Simulation}

The Monte Carlo module of the heterojunction compound 2-D finite element simulator $\mathrm{H} 2 \mathrm{~F}$ is described in detail elsewhere [15]. It is designed to simulate a wide range of recess gate compound FET's. A nonparabolic three-valley $(\Gamma, L, X)$ conduction band model is used for the III-V materials. The scattering mechanisms implemented include: ionized and neutral impurity, acoustic phonon, piezoelectric, optical phonon, and polar-optic scattering modes.

Quadrilateral finite elements are used in $\mathrm{H} 2 \mathrm{~F}$ to describe accurately the shape of the gate and the recess. The Poisson equation is solved self-consistently with the particle motion. The solution domain includes not only the semiconductor region but also the air and eventually the passivation surrounding the gate. The distribution of trapped charge on the pinning surface states before the transients is estimated from the results of drift-diffusion simulation [22]. It is assumed that during the transient the charge on the surface pinning states remains 
frozen. The recess shape and the surface conditions determine the part of the series resistances $R_{s 1}$ and $R_{s 2}$ associated with the recess, and influence also the charging resistance $R_{i}$. The recess and the gate shape influence also the gate-source $C_{\mathrm{gs}}$, gate-drain $C_{\mathrm{gd}}$, and the drain-source $C_{\mathrm{ds}}$ capacitances. These five passive components affect the RF performance of the simulated device.

The 2-D Monte Carlo treatment of transport is also essential to the accurate RF analysis. The transconductance $g_{m}$ is dominated by the velocity overshoot in the channel region and eventually in part of the recess region. $g_{m}$ is also influenced by the real space transfer of carriers outside the channel, which can be treated reliably only in Monte Carlo simulations. The output conductance $g_{\mathrm{ds}}$ is influenced both by the drain induced barrier lowering (DIBL), which is sensitive to the device geometry, and by the real space transfer.

The total transient terminal current required for the $y$ parameter extraction is a sum of the particle current $I_{p}(t)$ and the displacement current $I_{\text {disp }}(t)$. The particle current in Monte-Carlo simulations is usually estimated using the standard particle count method [23]. In this case, $I_{p}=n Q / \Delta t$, where $n$ is the number of super particles entering or leaving the given electrode during the time step $\Delta t$, and $Q$ is the charge of the super particles. Recalling the scales of the time step $\Delta t$, used at the beginning of the transient, it becomes clear that the stochastic fluctuations in the number count leads to substantial fluctuations in the estimated particle current. Usually a single particle entering/leaving the electrode during a typical time step of $1 \mathrm{fs}$ will introduce current fluctuation which is comparable to the average drain current in a FET in the normal mode of operation. The straight-forward use of the Gauss' law for calculating the displacement current $I_{\text {disp }}(t)$ also leads to significant current oscillations. The transient terminal currents can be statistically enhanced by employing more particles [12], [13].

In this work we use the Ramo-Shockley theorem to calculate the simultaneous contribution of all super particles involved in the EMC simulation to the total electrode current. The Ramo-Shockley theorem, formulated initially for the static case [24], [25], remains valid for time varying electrode potentials [26] and can be generalized to include electrons, holes, and generation and recombination processes [27]. However, in this work the hole distribution is frozen [15] and generation-recombination processes are not included in the simulation and therefore the approach outlined in [26] is adopted. Employing directly all moving particles, the Ramo-Shockley approach provides much smoother estimate for the total instantaneous current passing through a particular electrode. According to the Ramo-Shockley theorem the instantaneous transient current in electrode $i$ due to $N$ discrete moving charges within the device is given by

$$
I_{i}(t)=I_{i}^{\prime}(t)+I_{i}^{\prime \prime}(t)
$$

where the current $I_{i}^{\prime}(t)$ is solely contributed by the movement of the $N$ charged particles with fixed potentials at electrodes, and $I_{i}^{\prime \prime}(t)$ is the current induced due to the time-varying potentials of the electrodes through the capacitive coupling through the electrodes. The current $I_{i}^{\prime}(t)$ is given by

$$
I_{i}^{\prime}(t)=-\sum_{j=1}^{N} Q_{j} v_{j}(t) \cdot \nabla f_{i}
$$

where $Q_{j}$ is the charge of the super-particle, $v_{j}(t)$ is the velocity of the particle, and $f_{i}$ is the spatial distribution of the potential when a unit voltage is applied to electrode $i$, all other electrodes are grounded and all charges are removed from the device. In other words, $f_{i}$ is the solution of the Laplace equation $\nabla \cdot\left(\varepsilon \nabla f_{i}\right)=0$.

The current $I_{i}^{\prime \prime}(t)$ associated with the time varying potential is calculated from the capacitance matrix components $C_{\mathrm{ij}}$ associated with the electrodes of the simulated device. The Laplace equation is initially solved with all electrodes potential fixed to zero and then solved with the potential of each one electrode $j$ incremented by $\Delta V_{j}$. The capacitance matrix is then defined as

$$
C_{\mathrm{ij}}=\frac{\Delta Q_{i}}{\Delta V_{j}} .
$$

When a step perturbation $\Delta V_{j}$ of the terminal voltage is applied $I_{i}^{\prime \prime}(t)$ flows only during the first time step $\Delta t$ and is given by

$$
I_{i}^{\prime \prime}(t)=C_{\mathrm{ij}} \frac{\Delta V_{j}}{\Delta t} .
$$

The displacement current during the remaining part of the transient is related to the charges induced on the electrode by the moving particles in the device associated with the redistribution of the mobile charge and is accounted for by $I_{i}^{\prime}(t)$ [see (11)].

The $y$-parameter extraction procedure itself [(1), (2)] requires a small time step only immediately after the stepperturbation in order to resolve properly the inter-electrode capacitances. During the remaining part of the transient the time step may be larger than the time step $\Delta T$ used in the MC simulation in order to ensure self consistency between the particle-movement and field adjustment. Thus the transient currents obtained from the EMC simulation can be smoothed further before the extraction of the $y$-parameters by using a local averaging procedure. In the middle $k=\left(n^{\prime}-n\right) / 2$ of the interval between $n^{\prime}$ and $n$-th time steps the smoothed average current $I_{i}(k \cdot \Delta T)$ is given by

$$
I_{i}(t)=\frac{1}{n^{\prime}-n+1} \sum_{k=n}^{n^{\prime}} I_{i}(k \cdot \Delta T) .
$$

The maximum length of the averaging interval is restricted by the maximum frequency of interest according to $k \Delta T \ll$ $\left(2 \pi f_{h}\right)^{-1}$. In this study, the averaged traces of ten transients is used to reduce the uncertainty associated with the selection of timing of the initiation of the transient [12].

\section{RESUlTS AND DISCUSSION}

In order to illustrate the capabilities of the described complete EMC RF analysis we compare the result of our simulations with RF measurements of real pHEMT's fabricated 


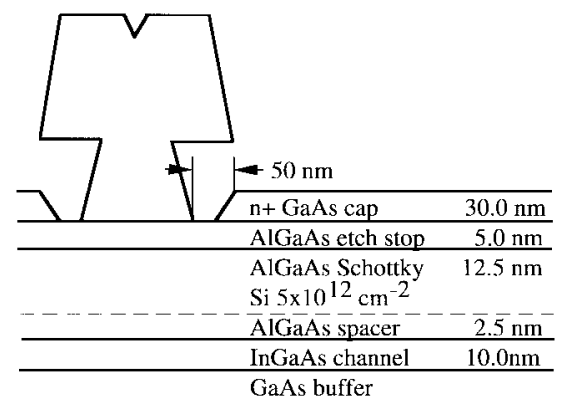

Fig. 4. The layer structure of the 120-nm gate length pHEMT.

in the Nanoelectronics Research Center of the University of Glasgow. The layer structure of the measured and simulated pHEMT is shown in Fig. 4. It comprises a 100-period superlattice grown on s.i. GaAs substrate followed by a $600-\mathrm{nm} \mathrm{GaAs}$ buffer. The strained $\operatorname{In}_{0.3} \mathrm{Ga}_{0.7}$ As channel is $10 \mathrm{~nm}$ thick and is separated from the $\mathrm{Si} \delta$-doping by a relatively thin $(2.5 \mathrm{~nm})$ $\mathrm{Al}_{0.3} \mathrm{Ga}_{0.7}$ As spacer. The $N_{s}=5 \times 10^{12} \mathrm{~cm}^{-2}$ effective $\delta$ doping is encapsulated between three and two monolayers GaAs. A 12.5-nm $\mathrm{Al}_{0.3} \mathrm{Ga}_{0.7}$ As Schottky layer, and 5-nm $\mathrm{Al}_{0.3} \mathrm{Ga}_{0.7}$ As recess etch stopper are grown on top of the $\delta$ doping. The overall separation between the channel and the gate is $21.5 \mathrm{~nm}$. A $30-\mathrm{nm}$ heavily doped $\left(N_{d}=4 \times 10^{18} \mathrm{~cm}^{-3}\right)$ GaAs cap layer screens the 2-DEG in the region between the gate and the contacts from the negative charge of electrons trapped on deep, acceptor type surface states. The channel length is $0.12 \mu \mathrm{m}$ with $50 \mathrm{~nm}$ gate recess on each side of the gate. The device has a channel width of $100 \mu \mathrm{m}$. The separation between the source and the drain contacts is $1 \mu \mathrm{m}$. Details of the gate recess region and the corresponding simulation domain are presented in Fig. 5(a) and (b).

The contact resistances and the external series resistance of the measurement equipment are incorporated in the EMC simulated $I_{d}-V_{d}$ characteristics using the procedure described in [20]. Since quantum effects and the associated intersubband scattering in the $\mathrm{In}_{0.3} \mathrm{Ga}_{0.7}$ As channel are not included in the current version of $\mathrm{H} 2 \mathrm{~F}$ the simulations overestimate velocity overshoot in the channel by approximately $15 \%$ and hence the drain current and the device transconductance.

The RF analysis is carried out at $V_{g}=-0.2 \mathrm{~V}$ and $V_{d}=1.5 \mathrm{~V}$ which corresponds to a typical operating point of the HEMT. The step changes in the gate and drain voltages are $\Delta V_{g}=200 \mathrm{mV}$ and $\Delta V_{d}=300 \mathrm{mV}$, respectively. The gate current following the gate voltage step extracted using the Ramo-Shockley procedure together with the standard particle count current and Gauss's integration approach are compared in Fig. 6 for the first 1 ps of the transient. The displacement current associated with the inter-electrode capacitances during the first time-step is several orders of magnitude higher and is not shown in this figure. The Ramo-Shockley trace shows dramatic noise improvement and contains instabilities mainly associated with plasma oscillations in the heavily doped regions of the device and eventually Gunn domain oscillations. The transient drain current corresponding to the same gate voltage step and calculated using the Ramo-Shockley procedure is given in Fig. 7. Prior to the extraction procedure, the gate and drain currents are further smoothed out using
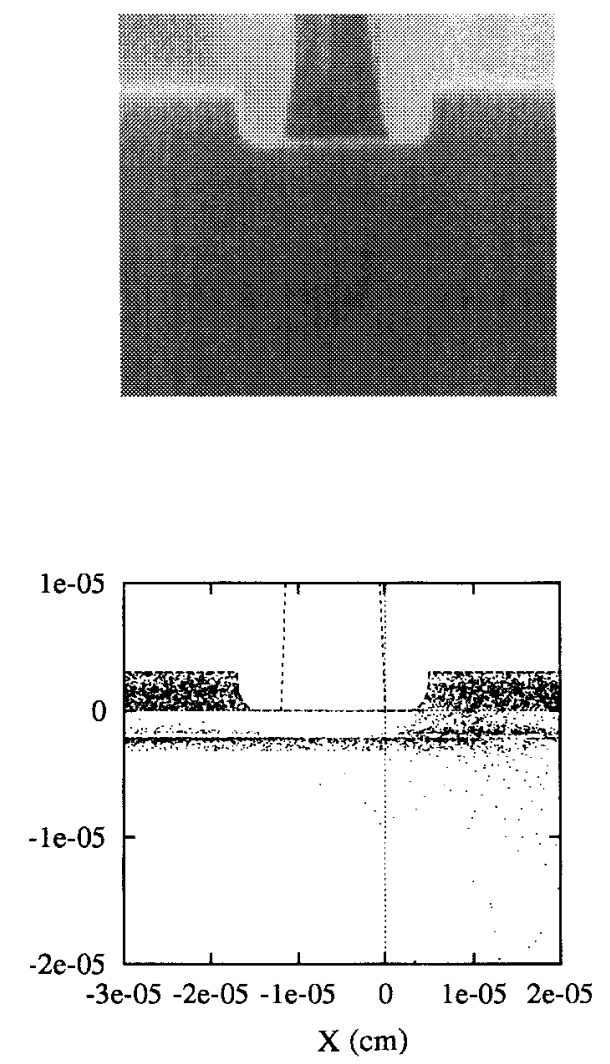

Fig. 5. SEM photograph of the 120-nm pHEMT gate and recess region together with the simulation domain.

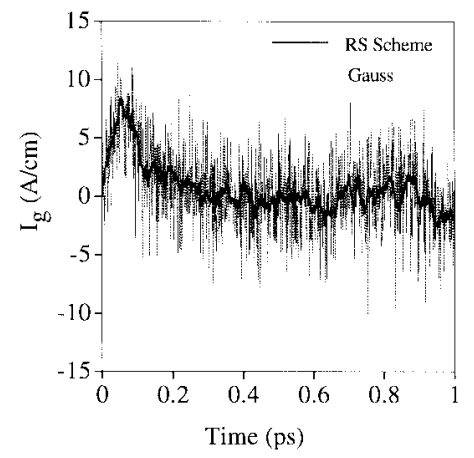

Fig. 6. Transient gate current following a change of $200 \mathrm{mV}$ in the gate potential $V_{g}=-0.2$ and $V_{d}=1.5 \mathrm{~V}$.

a temporal averaging procedure given by (14). The effect of the smoothing on the drain current transient is also shown in Fig. 7. The smoothing reduces the error of the numerical integration in (1) and (2) and results in more stable and smooth $y$-parameters.

The cutoff frequency of the simulated device estimated from the $y$-parameters $\mathbf{Y}^{\operatorname{sim}}$ is found to be $f_{T}^{\operatorname{sim}}=168 \mathrm{GHz}$. Then the $y$-parameters are transformed into $s$-parameters $\mathbf{S}^{\mathrm{sim}}$ from which the maximum frequency of oscillations of the simulated device was estimates to be $f_{\max }^{\operatorname{sim}}=631 \mathrm{GHz}$.

The source and drain resistance present in the simulated device were estimated to be $R_{s 1}=1.82 \Omega$ and $R_{d 1}=15.5 \Omega$, respectively. These resistances are subtracted from $\mathbf{Y}^{\text {sim }}$ through $z$-parameter transformation and the small-signal equivalent circuit components are extracted from the $y$-parameters of the 
TABLE I

Small-Signal Equivalent Circuit Components Extracted from Ten Current Traces and the Averaged Traces. Also Shown are the Experimental Equivalent Circuit Components

\begin{tabular}{|c|c|c|c|c|c|c|c|c|c|c|c|c|}
\hline Run \# & 1 & 2 & 3 & 4 & 5 & 6 & 7 & 8 & 9 & 10 & $\begin{array}{c}\text { Averaged } \\
\text { traces }\end{array}$ & Exp. \\
\hline$C_{g s}(\mathrm{fF})$ & 71 & 83.3 & 84 & 72 & 72.1 & 72 & 73 & 76.6 & 72.8 & 76.1 & 77.9 & 75.5 \\
\hline$C_{g d}(\mathrm{fF})$ & 16.4 & 12.5 & 12 & 16 & 16.4 & 16 & 16.4 & 12.5 & 16.5 & 16.5 & 14.2 & 8.7 \\
\hline$C_{d s}(\mathrm{fF})$ & 13.1 & 9 & 9 & 13.1 & 13.1 & 13 & 13.1 & 9 & 13.1 & 13 & 15.1 & 14.4 \\
\hline$g_{m o}(\mathrm{mS})$ & 87 & 89.4 & 89 & 87.6 & 87 & 86 & 85 & 85 & 86 & 93 & 87.6 & 67 \\
\hline$g_{d s}(\mathrm{nS})$ & 8.8 & 15 & 16 & 8.8 & 8.8 & 8.8 & 8.7 & 16 & 8.8 & 8.8 & 11.2 & 8.7 \\
\hline$R_{i}(\Omega)$ & 2.18 & 2.6 & 2.6 & 2.2 & 2.2 & 2.2 & 2.2 & 2.1 & 2.3 & 2.4 & 2.24 & 3.7 \\
\hline$\tau(\mathrm{ps})$ & 0.18 & 0.16 & 0.17 & 0.17 & 0.16 & 0.15 & 0.1 & 0.1 & 0.1 & 0.1 & 0.17 & 0.13 \\
\hline$f_{T}^{\text {real }}$ & 145 & 126 & 125 & 145 & 144 & 142 & 148 & 141 & 150 & 141 & 142 & 114 \\
\hline$f_{\max }^{\text {real }}$ & 166 & 141 & 140 & 166 & 167 & 163 & 171 & 167 & 172 & 160 & 164 & 150 \\
\hline
\end{tabular}

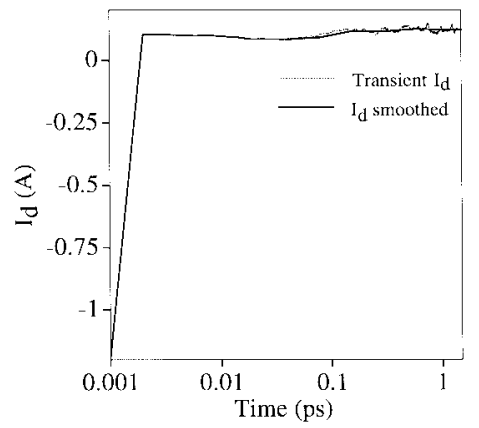

Fig. 7. Transient drain current following a change of $200 \mathrm{mV}$ in the gate potential together with the drain current smoothed using the local averaging procedure $V_{g}=-0.2$ and $V_{d}=1.5 \mathrm{~V}$.

intrinsic device $Y^{\text {int }}$. The extracted small-signal circuit components are plotted in Fig. 8(a)-(c) as functions of frequency. The virtual independence of the small-signal components on the frequency is a proof for the quality of the $y$-parameter extraction procedure.

The contact resistance present in the real device $R_{c}=3.5 \Omega$ and the gate resistance $R_{g}=5 \Omega$ typical for a pHEMT with 100- $\mu \mathrm{m}$ channel width were added to $\mathbf{Y}^{\mathrm{sim}}$ through $z$ parameter transformation to obtain the $y$-parameters of the "real" pHEMT $\mathbf{Y}^{\text {real }}$ from which the cutoff frequency of the "real" device was estimated to be $f_{T}^{\text {real }}=142 \mathrm{GHz}$. Then $\mathbf{Y}^{\text {real }}$ were transformed to $\mathbf{S}^{\text {real }}$ and the maximum frequency of oscillation of the "real" pHEMT was estimated to be $f_{\max }^{\text {real }}=164 \mathrm{GHz}$. The small-signal circuit components at $50 \mathrm{GHz}$ and figures of merit extracted from simulation are compared in Table I. The values are obtained from different traces where the transients are initiated at different times from the beginning of the EMC simulation. The table also shows the parameters obtained from ten averaged traces and the results of measurements on fabricated devices. The $20 \%$ difference between $f_{T}^{\text {real }}$ obtained from the simulations and the measured value is attributed to the overestimated transconductance.

To highlight the effect of the extrinsic parasitics on the device performance, we plot in Fig. 9 the dependence of $f_{\max }^{\text {real }}$ on the gate and the contact resistances. The results show that

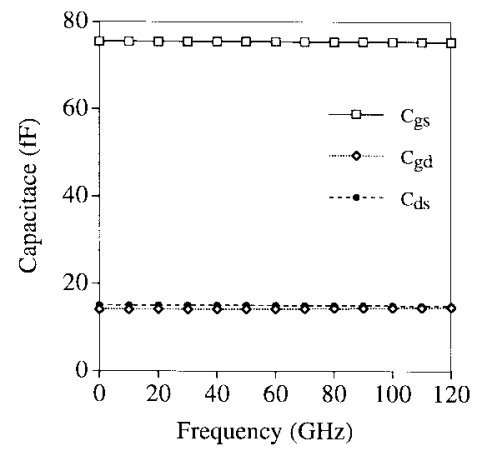

(a)

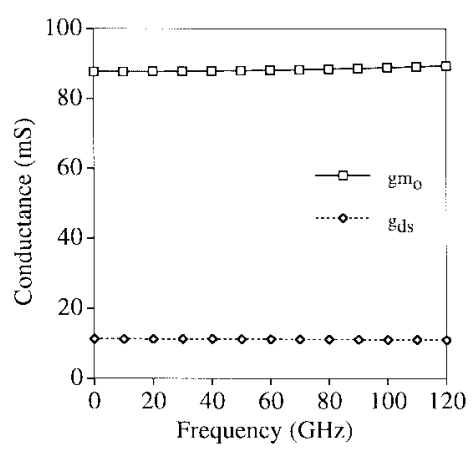

(b)

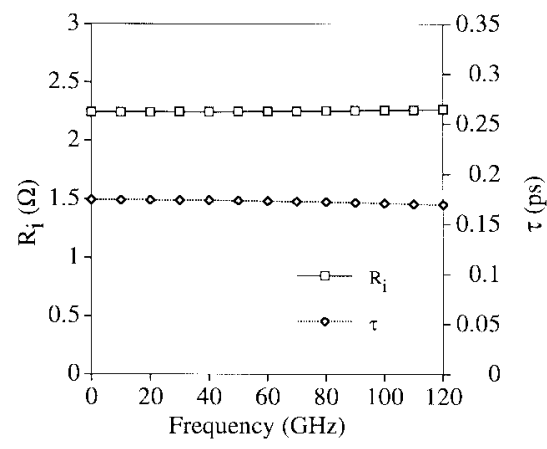

(c)

Fig. 8. Small-signal equivalent circuit components of 120 -nm gate length pHEMT as a function of frequency. 


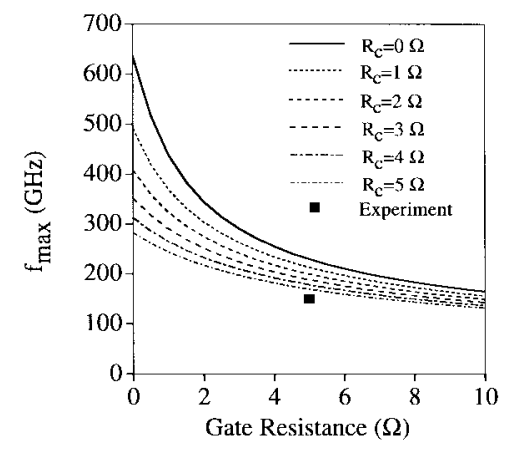

Fig. 9. Effect of the gate resistance and the source and drain contact resistance, $R_{c}$, on the maximum frequency of oscillation, $f_{\max }$., evaluated at $V_{g}=-0.2$ and $V_{d}=1.5 \mathrm{~V}$.

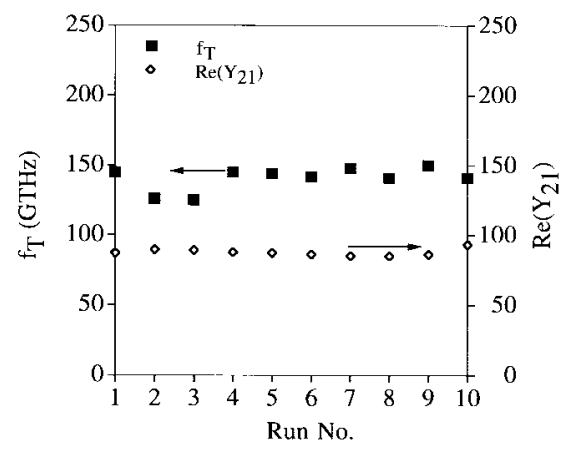

Fig. 10. $\operatorname{Re}\left(Y_{21}\right)$ and $f_{T}$ obtained from ten transients that are initiated at different moments after the steady state is established: $V_{g}=-0.2$ and $V_{d}=1.5 \mathrm{~V}$.

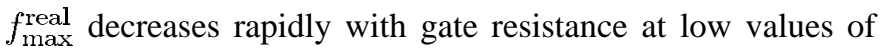
the contact resistances. A similar behavior is observed with the contact resistances at low gate resistance. Clearly, the highperformance potential of the pHEMT's is dramatically eroded by the extrinsic parasitics.

The fluctuation in the EMC terminal currents associated with plasma and Gunn domain oscillations introduce some degree of uncertainty in the extracted $y$-parameters. It could be expected that the values of the extracted $y$-parameters will depend to some extend on the choice of the moment at which the gate and drain voltage perturbations are applied. To investigate this uncertainty we compare the RF analysis results from ten transients initiated at different moments after the average steady-state conditions are established in the EMC calculations. The corresponding variation in the extracted $\operatorname{Re} Y_{21}$ is illustrated in Fig. 10. The fluctuation in $f_{T}^{\text {sim }}$ are mapped in Fig. 10. The extracted small-signal circuit components for the ten transients are summarized in Table I.

Finally, the frequency dependence of the minimum noise figure $\mathrm{NF}(f)$ of the intrinsic device calculated according to [21] is plotted in Fig. 11.

\section{CONCLUSION}

In this paper, a methodology for complete RF analysis of "real" compound FET's based on EMC simulation has been described. The device parasitics associated with surface effects

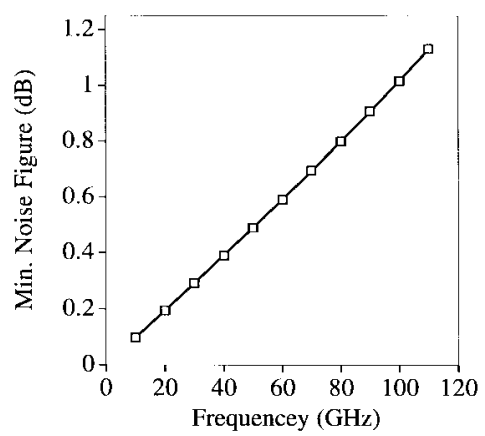

Fig. 11. Intrinsic noise figure, $\mathrm{NF}(f)$ of the 120-nm pHEMT extracted at $V_{g}=-0.2$ and $V_{d}=1.5 \mathrm{~V}$.

and the device geometry have been properly included in the $\mathrm{RF}$ analysis through our finite element EMC approach. The problems in the $y$-parameters extraction associated with the stochastic nature of the EMC current have been addressed. Statistical enhancement of the terminal transient currents has been achieved by explicit use of the Ramo-Shockley theorem in the case of time varying electrode potentials. The plasma type terahertz oscillations present in the simulations have been filtered out through a local averaging procedure. As a result, reliable $y$-parameters could be extracted for the whole range of operation voltages including saturation. The analytical procedure of extracting the equivalent circuit components of the simulated device [12] has been improved by excluding the source and drain access resistances, present in the simulation, from the $y$-parameters before extraction. The contact and the gate resistances have been includes in the $y$-parameters at the post-processing stage which allows estimation of important figures of merit such as $f_{T}^{\text {real }}$ and $f_{\max }^{\text {real }}$ of "real" devices.

The complete RF analysis has been applied in the simulation of real pHEMT's fabricated at Glasgow Nanoelectronics Research Center. The good agreement between the simulated and measured small-signal circuit components and performance figures of measures demonstrates the fruitfulness of our approach. The results of the post-processing analysis provide important insight into the performance potential of the intrinsic pHEMT's and the detrimental effect of the contact and the gate resistances.

The developed complete RF EMC analysis technique addresses practical RF device design issues in realistic device structures. It can be used also in the RF analysis of sub-0.1- $\mu \mathrm{m}$ $\mathrm{Si}$ and SiGe FET's where overshoot effects will dominate the channel transport and the EMC simulation will become essential.

\section{ACKNOWLEDGMENT}

The authors are thankful to J. Davies, S. Roy, and M. R. S. Taylor for the helpful discussions.

\section{REFERENCES}

[1] D. C. W. Lo, G. S. Dow, L. Yujiri, S. Chen, H. Wang, M. Biedenbender, M. Mussetto, and B. R. Allen, "Monolithic W-band seven-stage low noise amplifier/detector for radiometric imaging array application," Electron. Lett., vol. 33, pp. 1075-1077, 1994. 
[2] S. Babiker, N. Cameron, A. Asenov, and S. P. Beaumont, "New evidence for velocity overshoot in a 200-nm pseudomorphic HEMT," Microelectron. J., vol. 28, no. 8, pp. 785-793, 1996.

[3] Y. Awano, K. Tomizava, and N. Hashizume, "Principles of operation of short-channel gallium arsenide field-effect transistor determined by Monte Carlo method," IEEE Trans. Electron Devices, vol. ED-31, pp. 448-452, Apr. 1984

[4] G. M. Dunn, A. B. Walker, J. H. Jefferson, and D. C. Herbert, "Monte Carlo simulation of InP and GaAs MESFET's," J. Semicond. Sci. Technol., vol. 9, pp. 2123-2129, 1994.

[5] G. U. Jensen, B. Lund, T. A. Fjeldly, and M. Shur, "Monte Carlo simulation of short-channel heterostructure field-effect transistor," IEEE Trans. Electron Devices, vol. 38, pp. 840-851, Apr. 1991.

[6] K. W. Kim, H. Tian, and M. A. Litteljohn, "Analysis of delta-doped and uniformly doped AlGaAs/GaAs HEMT's by ensemble Monte Carlo simulation," IEEE Trans. Electron Devices, vol. 38, pp. 1737-1742, Aug. 1991.

[7] I. C. Kizilyally, M. Artaki, N. J. Shah, and A. Chandra, "Scaling properties and short-channel effects in submicrometer AlGaAs/GaAs MODFET's: A Monte Carlo study," IEEE Trans. Electron Devices, vol. 40, pp. 234-249, Feb. 1993.

[8] D. H. Park and K. F. Brennan, "Theoretical analysis of and $\mathrm{Al}_{0.15} \mathrm{Ga}_{0.85} \mathrm{As} / \mathrm{In}_{0.15} \mathrm{Ga}_{0.85}$ As pseudomorphic HEMT using ensemble Monte Carlo simulation," IEEE Trans. Electron Devices, vol. 36, pp. 1254-1263, July 1989

[9] "Monte Carlo simulation of $0.35-\mu \mathrm{m}$ gate-length GaAs and InGaAs HEMT's," IEEE Trans. Electron Devices, vol. 37, pp. 618-628, Mar. 1990.

[10] A. Asenov, S. Babiker, N. Cameron, M. R. S. Taylor, and S. P. Beaumont, "Impact of gate recess offset on pseudomorphic HEMT performance: A simulation study," in Proc. European Solid State Device Res. Conf., G. Baccarani and M. Rudan, Eds. Bologna, Italy: Editions Frontiers, 1996, pp. 1017-1020.

[11] C. Moglestu, "A self-consistent Monte Carlo particle model to analyze semiconductor micro-components of any geometry," IEEE Trans. Computer-Aided Design, vol. CAD-5, pp. 326-345, Apr. 1986.

[12] T. Gonzàlez and D. Pardo, "Monte Carlo determination of the intrinsic small-signal equivalent circuit of MESFET's," IEEE Trans. Electron Devices, vol. 42, pp. 605-611, Apr. 1995.

[13] T. Gonzàlez, D. Pardo, L. Varani, and L. Reggiani, "Monte Carlo analysis of the behavior and spatial origin of electronic noise in GaAs MESFET's," IEEE Trans. Electron Devices, vol. 42, pp. 991-998, May 1995.

[14] A. Abou-Elnour and K. Schünemann, "Noise calculations of submicrometer MESFET's by using 2-D physical simulators," J. Electron. Commun., vol. 50, no. 5, pp. 334-342, 1996.

[15] S. Babiker, A. Asenov, J. R. Barker, and S. P. Beaumont, "Finite element Monte Carlo simulation of recess gate compound FET's," Solid State Electron., vol. 39, pp. 629-635, 1996.

[16] S. Laux, "Techniques for small-signal analysis of semiconductor devices," IEEE Trans. Electron Devices, vol. ED-32, pp. 2028-2037, Oct. 1985.

[17] M. Wojtowicz, R. Lai, D. Streit, G. I. Ng, T. R. Block, K. L. Tan, P. H. Liu, A. K. Freudenthal, and R. M. Dia, " $0.1-\mu \mathrm{m}$ graded InGaAs channel InP HEMT with $305 \mathrm{GHz} f_{T}$ and $340 f_{\max }$," IEEE Electron Device Lett., vol. 15, pp. 477-479, Nov. 1994.

[18] M. Berroth and R. Bosc, "Broad-band determination of the FET smallsignal equivalent circuit," IEEE Trans. Microwave Theory Tech., vol. 38, pp. 891-895, July 1990 .

[19] K. Shirakawa, H. Oikawa, T. Shimura, Y. Kawasaki, Y. Ohashi, T. Saito, and Y. Daito, "An approach to determining an equivalent circuit for HEMT's," IEEE Trans. Microwave Theory Tech., vol. 43, pp. 499-503, Mar. 1995.

[20] S. Babiker, A. Asenov, N. Cameron, and S. P. Beaumont, "Simple approach to include external resistances in the Monte-Carlo simulation of MESFET's and HEMT's," IEEE Trans. Electron Devices, vol. 43, pp. 2032-2034, Nov. 1996.

[21] M. Pospieszalski, "Modeling of noise parameters of MESFET's and MODFET's and their frequency and temperature dependence," IEEE Trans. Microwave Theory Tech., vol. 37, pp. 1340-1350, Sept. 1989.

[22] A. Asenov, D. Reid, J. R. Barker, N. Cameron, and S. P. Beaumont, "Finite element simulation of recess gate MESFET's and HEMT's.: The Simulator H2F," in Simulation of Semiconductor Devices and Processes, S. Selberherr, H. Stippel, and E. Strasser, Eds. Wien, Germany: Springer-Verlag, 1993, vol. 5, pp. 265-268.

[23] C. Moglestue, Monte Carlo Simulation of Semiconductor Devices. London, U.K.: Chapman \& Hall, 1993.
[24] S. Ramo, "Currents induced by electron motion," in Proc. IRE, 1939, vol. 27, p. 548

[25] W. Shockley, "Current to conductors induced by a moving point charge," J. Appl. Phys., vol. 9, p. 635, 1938.

[26] H. Kim, H. Min, T. Tang, and Y. Park, "An extended proof of the Ramo-Shockley theorem," Solid State Electron., vol. 34, no. 11, pp. 1251-1253, 1992.

[27] P. D. Yoder, K. Gartner, and W. Fichtner, "A generalized Ramo-Schockley theorem for classical to quantum transport at arbitrary frequencies," J. Appl. Phys., vol. 79, 1996.

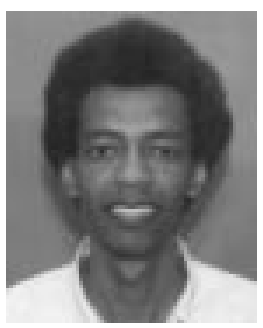

Sharief Babiker received the B.Sc. degreee in electrical engineering from Khartoum University, Sudan, in 1984, and the Ph.D. degree from Glasgow University, Glasgow, Scotland, U.K., in 1994 for work on the theory and modeling of singleelectronic devices and systems.

From 1985 to 1990 , he was with the Electrical Engineering Department, Khartoum University. His current research interests concentrate on the simulation and modeling of FET's with emphasis on submicron gate length InGaAs channel pHEMT's for RF applications. Currently, he is with the Nanoelectronics Research Centre, University of Glasgow.

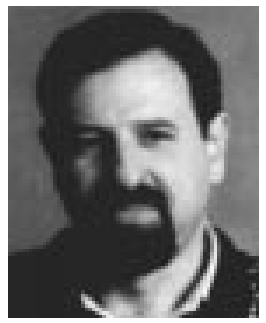

Asen Asenov has had ten years industrial experience as a head of the Process and Device Modeling Group in IME, Sofia, Bulgaria, developing one of the first integrated process and device CMOS simulators IMPEDANCE. He was Visiting Professor in the Physics Department of Technical University, Munich, Germany, and is currently a Reader in the Department of Electronics and Electrical Engineering, Glasgow University, Glasgow, Scotland, U.K. As a leader of the Device Modeling Group and Academic Director of the Process and Device Simulation Center at Glasgow University, he leads the development of 2-D and 3-D device simulators and their application in the design of FET's, SiGe MOSFET's, and IGBT's. He has over 110 publications in process and device modeling and simulation, semiconductor device physics, "atomistic" effects in ultrasmall devices, and parallel computing.

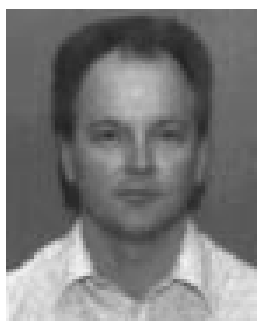

Nigel Cameron received the B.Sc. degree from the University of Bath, U.K., in 1984.

He joined British Telecom's Research Laboratories, Ipswich, U.K., to work on plasma process research and development for silicon fabrication. In March 1988, he moved to the Nanoelectronics Research Center, the University of Glasgow, Glasgow, Scotland, U.K., where his responsibilities include the development of ultrafast transistors in compound semiconductors and their integration into manufacturable processes for millimeter-wave integrated circuits (MMIC's). His interests extend across a broad range of semiconductor technologies encompassing materials and device physics; circuit design and test; manufacturing; and $\mathrm{dc}$ and high-frequency characterization. He is the author or coauthor of more than 35 technical papers. 


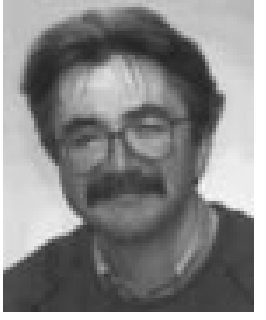

Steven P. Beaumont received the M.A. and Ph.D. degrees from the University of Cambridge, Cambridge, U.K., in 1975 and 1979, respectively.

He has been with the Department of Electronics and Electrical Engineering, the University of Glasgow, Glasgow, Scotland, U.K., since 1978. He became Head of Department in 1995 and he convenes the Nanoelectronics Research Center's Management Committee. His research interests lie in the field of nanometer-scale fabrication and its application to electronic and optoelectronic devices. He has over 100 publications on electron beam nanolithography, dry etching, short-gate III-V based transistors, quantum transport devices, the optical properties of quantum dots, and single-electron devices. Latterly, he has become involved with the issue of manufacturability of millimeter-wave circuits and the use of nanometer-scale fabrication techniques coupled with technology-based device simulations to forecast performance and yield with the minimum of process iterations.

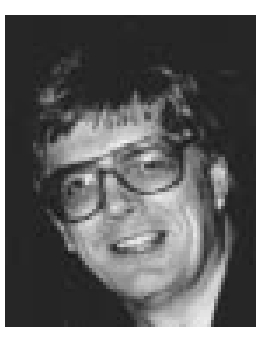

John R. Barker is Professor of Electronics in the Department of Electronics and Electrical Engineering, University of Glasgow, Glasgow, Scotland, U.K. He has a long standing interest in computational methods, device modeling, and transport theory. From 1970 to 1985 , he was a member of the Theory Group in the Department of Physics, University of Warwick, U.K., aside from the period 1978-1979, when he worked at IBM T. J. Watson Laboratory, North Texas State University, and Colorado State University. From 1987 to 1989, he was Academic Director of the IBM UK/Glasgow University Kelvin Project on Numerically Intensive Parallel Computing. Currently, he is Academic Director of the Parallel Processing Center, University of Glasgow. 